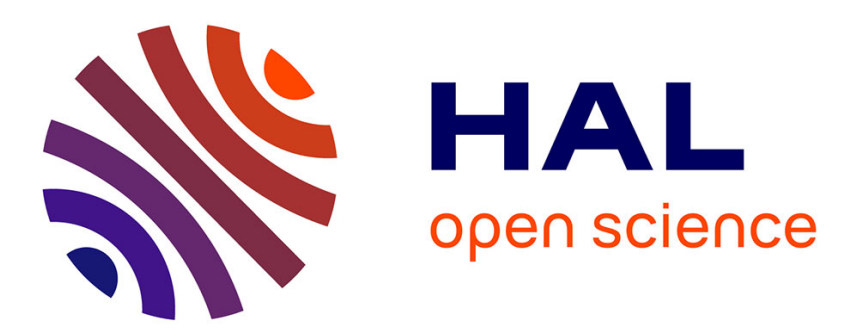

\title{
Lyapunov techniques for stabilization of switched linear systems of conservation laws
}

Pierre-Olivier Lamare, Antoine Girard, Christophe Prieur

\section{To cite this version:}

Pierre-Olivier Lamare, Antoine Girard, Christophe Prieur. Lyapunov techniques for stabilization of switched linear systems of conservation laws. CDC 2013 - 52nd IEEE Conference on Decision and Control, Dec 2013, Florence, Italy. pp.448-453, 10.1109/CDC.2013.6759922 . hal-00926379

\section{HAL Id: hal-00926379 \\ https://hal.science/hal-00926379}

Submitted on 9 Jan 2014

HAL is a multi-disciplinary open access archive for the deposit and dissemination of scientific research documents, whether they are published or not. The documents may come from teaching and research institutions in France or abroad, or from public or private research centers.
L'archive ouverte pluridisciplinaire HAL, est destinée au dépôt et à la diffusion de documents scientifiques de niveau recherche, publiés ou non, émanant des établissements d'enseignement et de recherche français ou étrangers, des laboratoires publics ou privés. 


\title{
Lyapunov techniques for stabilization of switched linear systems of conservation laws
}

\author{
Pierre-Olivier Lamare, Antoine Girard and Christophe Prieur
}

\begin{abstract}
In this paper, the exponential stability in $L^{2}$ norm is investigated for a class of switched linear systems of conservation laws. The state equations and the boundary conditions are both subject to switching. We consider the problem of synthesizing stabilizing switching controllers. By means of Lyapunov techniques, three control strategies are developed based on steepest descent selection, possibly combined with a hysteresis and a low-pass filter. Some numerical examples are considered to illustrate our approach and to show the merits of the proposed strategies.
\end{abstract}

\section{INTRODUCTION}

Physical networks may be represented by hyperbolic partial differential equations in one space dimension, yielding systems of conservation laws or balance laws. Among the different networks, we have in mind the hydraulic networks (see [3], [4]), road traffic networks (see [12]), or gas pipeline networks (see [7]). Due to the range of those applications in the engineering field, an important research on the theorical aspects in modelling and control of such systems is made. The present paper deals with switched linear systems of conservation laws, and their control.

In such systems, some parameters may evolve in time. For example, it can be the boundary conditions in an open channel controlled by mobile spillways, or the state equations of a road where the speed limitation changes during the time. Of course, the state equations and the boundary conditions can both be subject to abrupt changes. Therefore a switched system occurs: a system where there is both a continuous dynamic (given by the state equation) and a discrete dynamic (given by a switching signal).

Some results are available in literature for the stability and stabilizability of switched hyperbolic systems of balance laws or conservation laws. In [16] some sufficient conditions for the asymptotic stability are stated, uniformly with respect to a class of switching signals. In [1], a result of stability is given under an arbitrary switching signal using the propagation of the solution along the characteristics. For switched systems governed by semigroups of linear evolution operators and their stability under an arbitrary switching signal, see [11]. In [8] a star-shaped network with a central node, and the wave equation governing on each edge, is

P.-O. Lamare and A. Girard are with Laboratoire Jean Kuntzmann, Université de Grenoble, BP 53, 38041 Grenoble, France. pierre-olivier.lamaredimag.fr, antoine.girardeimag. fr.

C. Prieur is with Department of Automatic Control, Gipsa-lab, 11 rue des Mathématiques, BP 46, 3840238402 Saint Martin d'Hères Cedex, France. christophe.prieurdgipsa-lab. fr

This work has been partially supported by the LabEx PERSYVAL-Lab ANR-11-LABX-0025 and by PHC TOURNESOL FR 2013. studied. It is shown that a switching feedback stabilization at the exterior end of the edge is effective under some sufficient conditions. Moreover it is proved that the problem has solutions under the proposed switching rule. The wellposedness issue and the dependence of the solutions on the initial data for a networked transport systems defined on directed graph is explored in [9], while an analysis based on functions of bounded variation in an extended way is used in [10] to prove well-posedness for a quasilinear system of first-order hyperbolic PDEs. In this last two references the switching modeling is view as a tool for systems for which different time and spatial scales interact.

An approach commonly used to study the stability and to design the boundary control of system of conservation laws is the Lyapunov analysis [2], [3]. In this paper we use this approach (as in [16]) to design three swiching rules for our switched hyperbolic system of conservation laws. We show that, under a sufficient condition, global exponential stability in $L^{2}$-norm is obtained with these switching rules. The sufficient condition takes the form of a linear matrix inequality (LMI). Hence the verification of this condition is expressed as an optimization program, which can be solved numerically. Moreover an upper bound on the speed of convergence is given, and it is possible to numerically optimize it in the three cases proposed in this paper.

The paper is organized as follows. In Section II, we introduce the class of switched linear hyperbolic systems of conservation laws considered in this paper. In Section III, the common Lyapunov function used in our analysis is introduced, and some preliminary results are derived, then the three switching rules are proposed, with the corresponding results obtained with them. Finally in Section IV, an example is studied to illustrate our main results.

Due to space limitation, the proofs of all results are omitted.

Notation. The set $\mathbb{R}^{+}$is the set of nonnegative real numbers. The set of square real matrices of dimension $n$ is denoted by $\mathbb{R}^{n \times n}$. Given a matrix $A$, the transpose of the matrix $A$ is denoted by $A^{\top}$. Given $n$ real numbers $a_{1}, \ldots, a_{n}$ the matrix $A \in \mathbb{R}^{n \times n}$ with these numbers on the diagonal is denoted by $\operatorname{diag}\left(a_{1}, \ldots, a_{n}\right)$. The identity matrix of dimension $n$ is denoted by $I_{n}$, while the zero matrix of dimension $n$ is denoted by $0_{n}$. For a symmetric matrix $A \in \mathbb{R}^{n \times n}, A$ being positive definite is denoted $A>0$, while $A$ being positive semi-definite is denoted $A \geq 0$. The usual Euclidian norm in $\mathbb{R}^{n}$ is denoted by |.|. The set of functions $y:[0,1] \rightarrow \mathbb{R}^{n}$ such that $|y|_{L^{2}([0,1])}^{2}=\int_{0}^{1}|y(x)|^{2} d x<\infty$, is denoted by $L^{2}([0,1])$. Given a continuous function $y$ 
on an open interval $\left(x_{1}, x_{2}\right)$ and taking value in $\mathbb{R}^{n}$, the infinity norm is denoted by $|y|_{L^{\infty}}=\inf \{C ;|y| \leq C$ a.e. $\}$. The restriction of a function $y:[0,1] \rightarrow \mathbb{R}^{n}$ on an open interval $\left(x_{1}, x_{2}\right) \subset[0,1]$ is denoted by $y_{\mid\left(x_{1}, x_{2}\right)}$.

\section{Problem Formulation}

We are concerned with $n \times n$ linear hyperbolic systems of conservation laws of the form:

$$
\left\{\begin{array}{c}
\partial_{t} y(t, x)+\Lambda_{\sigma(t)} \partial_{x} y(t, x)=0, \\
t \in \mathbb{R}^{+}, x \in(0,1), \\
y(t, 0)=G_{\sigma(t)} y(t, 1), \quad t \in \mathbb{R}^{+}, \\
y(0, x)=y^{0}(x) \quad x \in[0,1],
\end{array}\right.
$$

where $y:[0,1] \times \mathbb{R}^{+} \rightarrow \mathbb{R}^{n}, \sigma: \mathbb{R}^{+} \rightarrow \mathcal{I}$ where $\mathcal{I}=$ $\{1, \ldots, N\}$ is a finite set of indices. The function $\sigma$ is called switching signal of the system. The times of discontinuities of $\sigma, 0<t_{1}<t_{2}<\ldots$ are called switching times.

For all $i \in \mathcal{I}, G_{i}$ belongs to $\mathbb{R}^{n \times n}, \Lambda_{i}$ is a diagonal positive definite matrix in $\mathbb{R}^{n \times n}$ i.e. $\Lambda_{i}=\operatorname{diag}\left(\lambda_{i, 1}, \ldots, \lambda_{i, n}\right)$ for suitable positive values $\lambda_{i, 1} \ldots \lambda_{i, n}$.

Now we explicit the type of function $y$ that we consider for the solution of system (1).

Definition 2.1: A piecewise continuous function $y$ : $[0,1] \rightarrow \mathbb{R}^{n}$ is a continuous function on $[0,1]$ except maybe on a finite number of points $0=x_{0}<x_{1}<\cdots<x_{p}=1$ such that for all $l \in\{0, \ldots, p-1\}$ there exists $y_{l}$ continuous on $\left[x_{l}, x_{l+1}\right]$ and $y_{l}=y_{\mid\left(x_{l}, x_{l+1}\right)}$. The set of all piecewise continuous functions is denoted by $C_{p w}([0,1])$.

Let

$$
\begin{aligned}
&\|y\|=\max \left\{\max _{l \in\{0, \ldots, p\}}\left\{\left|y\left(x_{l}\right)\right|\right\},\right. \\
&\left.\max _{l \in\{0, \ldots, p-1\}}\left\{\left|y_{\mid\left(x_{l}, x_{l+1}\right)}\right|_{L^{\infty}}\right\}\right\} .
\end{aligned}
$$

One easily verifies that $\|$.$\| is the classical norm \mathcal{L}^{\infty}$ for the space $C_{p w}([0,1])$. Now we assume that the initial data $y^{0}$ lies in $C_{p w}([0,1])$. The trace of $y \in C_{p w}([0,1])$ is well defined. Indeed the application $T:\left(C_{p w}([0,1]),\|\|.\right) \rightarrow\left(\mathbb{R}^{n},|\cdot|\right)$, $T: y \mapsto y(1)$ is linear and continuous. In this paper it is assume that the function $y(t, \cdot)$ lies in $C_{p w}([0,1])$ for all $t \geq$ 0 , so the number of discontinuities in the solution is supposed to grow but staying finite along the time. In other words, the results presented in this paper are valid as long as no Zeno behaviors occur (see [14] for example for a definition of this phenomena).

The aim of this work is to design an output controller. It will be a switching rule which depends only on the measurement at the boundary of the domain, in order to stabilize the system in the following sense:

Definition 2.2: Given a switching signal, system (1) is said to be globally exponentially stable (in $L^{2}$-norm), if there exist positive constants $c$ and $\alpha$ such that the solution of (1) exists, is unique and satisfies the inequality

$$
|y(t, \cdot)|_{L^{2}([0,1])} \leq c e^{-\alpha t}\left|y^{0}\right|_{L^{2}([0,1])},
$$

for all $t \geq 0$.
Now, we will make clear the notion of switching signal dependent of the measurement at the boundary. In general, the state of a hyperbolic system of conservation laws is measured at the boundaries of the domain thanks to a set of sensors. In our case the system is only observed at the point $x=1$ at any time because of the positive values of velocities $\lambda_{i, 1} \ldots \lambda_{i, n}$. The sensor values are defined as

$$
w(t)=y(t, 1) .
$$

The output function is defined as soon as $y(t, \cdot) \in$ $C_{p w}([0,1])$, since the trace of a function in $C_{p w}([0,1])$ is well-defined (see above).

According to the sensor values, the system (1) will evolve in a particular mode. Thus the switching rule is denoted by

$$
\begin{aligned}
\sigma[w]: & \mathbb{R}^{+} \rightarrow \mathcal{I} \\
& t \mapsto \sigma[w](t) .
\end{aligned}
$$

Moreover the switching rule is a causal decision: the switching rule does not depend on the future value of the system state but only on current and past values. Mathematically it can be written as follows:

$w(t)=w^{\prime}(t), \forall t \in[0, \tau] \Rightarrow \forall t \in[0, \tau], \sigma[w](t)=\sigma\left[w^{\prime}\right](t)$.

To summarize, the process evolves in a mode, a sensor measures the state $w(t)$ of the process at the boundary, then depending on this value, a switching rule imposes the mode in which the system must evolve afterwards.

In order to design this switching rule, we will perform an analysis based on Lyapunov techniques.

Let us conclude this section with two remarks.

Remark 2.3: In system (1), the matrices $\Lambda_{i}$ are diagonal positive definite. This assumption is made only for the sake of simplicity in our analysis. Indeed we can consider more general diagonal matrices for $\Lambda_{i}$. Suppose that there exists $m>0$ such that for all $i \in \mathcal{I}, \Lambda_{i}$ is a diagonal matrix satisfying $\Lambda_{i}=\operatorname{diag}\left(\lambda_{i, 1}, \ldots, \lambda_{i, n}\right)$ with $\lambda_{i, k}>0$ for $k \in$ $\{1, \ldots, m\}$ and $\lambda_{i, k}<0$ for $k \in\{m+1, \ldots, n\}$. The matrices $\Lambda_{i}$ are written as $\Lambda_{i}=\left(\begin{array}{cc}\Lambda_{i}^{+} & 0_{n-m, m} \\ 0_{m, n-m} & \Lambda_{i}^{-}\end{array}\right)$, where $\Lambda_{i}^{+}=$ $\operatorname{diag}\left(\lambda_{i, 1}, \ldots, \lambda_{i, m}\right)$ and $\Lambda_{i}^{-}=\operatorname{diag}\left(\lambda_{i, m+1}, \ldots, \lambda_{i, n}\right)$ are respectively diagonal positive definite matrices and diagonal negative definite matrices. We introduce the notations $y^{+}=\left(y_{1}, \ldots, y_{m}\right)^{\top}, y^{-}=\left(y_{m+1}, \ldots, y_{n}\right)^{\top}$, such that $y=\left(y^{+}, y^{-}\right)^{\top}$. The system in its general form is

$$
\left\{\begin{array}{c}
\partial_{t} y(t, x)+\Lambda_{\sigma[w](t)} \partial_{x} y(t, x)=0 \\
t \in \mathbb{R}^{+}, x \in(0,1) \\
\left(\begin{array}{c}
y^{+}(t, 0) \\
y^{-}(t, 1)
\end{array}\right)=G_{\sigma[w](t)}\left(\begin{array}{l}
y^{+}(t, 1) \\
y^{-}(t, 0)
\end{array}\right) \\
y(0, x)=y^{0}(x)
\end{array}\right.
$$

where $G_{i}=\left(\begin{array}{c}G_{i}^{++} G_{i}^{+-} \\ G_{i}^{-+} G_{i}^{--}\end{array}\right)$, such that $G_{i}^{++}, G_{i}^{+-}, G_{i}^{--}$ and $G_{i}^{-+}$are matrices respectively in $\mathbb{R}^{(n-m) \times(n-m)}$, $\mathbb{R}^{(n-m) \times m}, \mathbb{R}^{m \times(n-m)}$ and $\mathbb{R}^{m \times m}$. By the change of variable $z(t, x)=\left(y^{+}(t, x), y^{-}(t, 1-x)\right)^{\top}$ we obtain a new system in the same form as (1). 
As in [16] only the case where the number of negative and positive eigenvalues of $\Lambda_{i}$ are the same, for all $i \in \mathcal{I}$, is considered.

Remark 2.4: An attractive result to analyze the stability of the systems without switching is given in [2]. With the same notation of [2], for all matrices $M \in \mathbb{R}^{n \times n}$ we denote

$$
\rho_{1}(M)=\inf \left\{\left\|\Delta M \Delta^{-1}\right\|, \Delta \in \mathcal{D}_{n,+}\right\},
$$

where $\mathcal{D}_{n,+}$ denotes the set of diagonal positive definite matrices in $\mathbb{R}^{n \times n}$. Thanks to Proposition 3.2 of [2] one has that, for all matrices $M \in \mathbb{R}^{n \times n}$,

$$
\rho_{1}(M) \leq \rho(|M|),
$$

where $\rho(|M|)$ denotes the spectral radius of the matrix whose elements are the absolute values of the elements of $M$. Theorem 2.3 of [2] gives the following result: if $\rho_{1}(G)<1$ then the system (1) without switching is stable, and a fortiori if $\rho(|G|)<1$ the same result is obtained. We will use this result for the example of Section IV below.

\section{Stabilization}

\section{A. Lyapunov function}

In this section, preliminary results on Lyapunov functions are derived. Following [2], the candidate Lyapunov function that is considered in this paper is written as, for all $y \in$ $L^{2}([0,1])$,

$$
V(y)=\int_{0}^{1} y(x)^{\top} Q y(x) e^{-\mu x} d x, \mu>0,
$$

for a given diagonal positive definite matrix $Q \in \mathbb{R}^{n \times n}$. For the system with any constant control input $\sigma(t)=\bar{i}$, it is shown in [2] that $\rho_{1}\left(G_{\bar{i}}\right)<1$ is a sufficient condition for the existence of a Lyapunov function of the form (7) that stabilizes system (1).

Let $y$ be a solution of system (1). We shall denote in the following

$$
\forall t \geq 0, V=V(y(t, \cdot)) \text { and } \dot{V}=\frac{d}{d t} V(y(t, \cdot)) .
$$

In the sequel, we denote by $\lambda$ the smallest eigenvalue of the matrices $\Lambda_{i}$, for all $i \in \mathcal{I}$,

$$
\lambda=\min _{i \in\{1, \ldots, N\}}\left(\min _{j \in\{1, \ldots, n\}} \lambda_{i, j}\right) .
$$

With these notations at hand we are able to state our first lemma, giving an inequality for the time derivative of $V$ along the solutions of the switched system of conservation laws (1). This inequality will be useful to design the switching rules, and to give the proof of stability of the system (1) with them.

Lemma 3.1: The time derivative of the candidate Lyapunov function $V$ along the solution of (1) satisfies

$$
\dot{V} \leq-2 \alpha V+q_{i}(w(t)),
$$

where $i \in \mathcal{I}$ is the active mode at time $t, q_{i}(w(t))=$ $w(t)^{\top}\left[G_{i}^{\top} Q \Lambda_{i} G_{i}-Q \Lambda_{i} e^{-\mu}\right] w(t)$ and $\alpha=\frac{1}{2} \mu \lambda$.

Remark 3.2: Lemma 3.1 still holds when $Q$ is a symmetric, positive definite matrix such that $Q$ and $\Lambda_{i}$ commute for all $i \in \mathcal{I}$.

\section{B. Switching strategies}

1) Argmin: In this section, we consider the closed-loop dynamics of the switched system of conservation laws (1) when using output definition (5).

Following the idea developed in [6] and recalling the notation $q_{i}$ in Lemma 3.1 we define the memoriless switching rule

$$
\sigma[w](t)=\underset{i \in\{1, \ldots, N\}}{\arg \min } q_{i}(w(t)) .
$$

The idea of the argmin switching rule is to choose the mode which optimizes the decrease of the Lyapunov function at any time. So we need a condition which ensures that there always exists a mode for which the system is decreasing. To this end, let us define the simplex

$$
\Gamma:=\left\{\gamma \in \mathbb{R}^{N} \mid \sum_{i=1}^{N} \gamma_{i}=1, \gamma_{i} \geq 0\right\} .
$$

To study the stability of the switched system of conservation laws (1) we need the following

Assumption 3.3: There exist $\gamma \in \Gamma$, a diagonal definite positive matrix $Q$ and a parameter $\mu>0$ such that

$$
\sum_{i=1}^{N} \gamma_{i}\left(G_{i}^{\top} Q \Lambda_{i} G_{i}-e^{-\mu} Q \Lambda_{i}\right) \leq 0 .
$$

Remark 3.4: An important issue is the numerical computation of $\gamma \in \Gamma, \mu>0$ and of a diagonal positive definite matrix $Q$ such that (13) holds. In the case where $N=2$ a solution consists in performing a line search over the parameters $\gamma$ and $\mu$ and, for each pair $(\gamma, \mu)$, to solve a convex problem in the variables $Q$ written in terms of the LMI (13). This can be done numerically in polynomial time. Otherwise the problem is bilinear and the numerical verification of Assumption 3.3 can be quite complex. $\quad$

Remark 3.5: Denoting $P_{i}=\Lambda_{i}^{\frac{1}{2}} G_{i} \Lambda_{i}^{-\frac{1}{2}}$, the condition (13) is rewritten as $\sum_{i=1}^{N} \gamma_{i}\left(P_{i}^{\top} Q P_{i}-e^{-\mu} Q\right) \leq 0$. As noted in [6] this last condition implies that the convex combination $P_{\gamma}=\sum_{i=1}^{N} \gamma_{i} P_{i}$ is asymptotically stable. Thus to check the existence of a matrix $Q$ and a positive parameter $\mu$ such that Assumption 3.3 holds, it is useful to verify the existence of a stable convex combination of matrices $P_{i}$. The converse does not hold in general: the existence of a stable $P_{\gamma}$ does not imply that Assumption 3.3 holds.

Assumption 3.3 implies that there always exists a mode $i \in \mathcal{I}$ such that $q_{i}(w(t)) \leq 0$. Thus we can give our first result of exponential stability of the system (1) with the argmin switching rule.

Theorem 3.6: Under Assumption 3.3, system (1) with switching rule (11) is globally exponentially stable. More precisely, letting $V$ and $\alpha$ as in Lemma 3.1, (3) holds along the solutions of (1) with (11).

2) Argmin with hysteresis: The first result shows that under Assumption 3.3, the switched system of conservation laws (1) with the argmin switching rule is globally exponentially stable. The limitation of this rule is a possible fast switching behavior (see Table I in Section IV for 


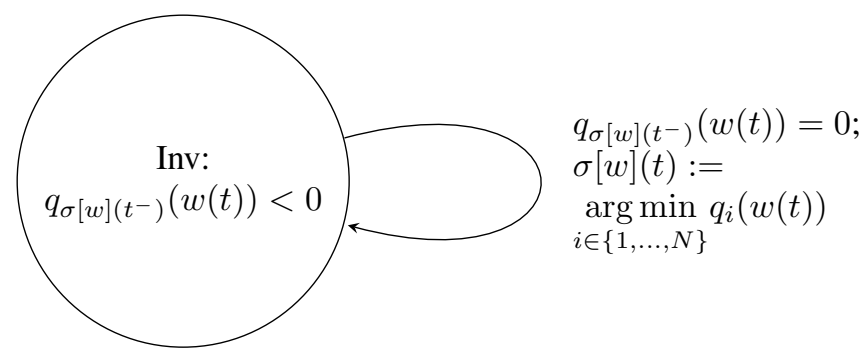

Fig. 1. Argmin with hysteresis automaton.

an example). Unfortunately, in many applications this is undesirable. So the goal is to use strategies to slow down the switching. The first one is the hysteresis strategy.

For all $t>0$ we denote by $\sigma[w]\left(t^{-}\right)$the limit from the left of $t$ of the value of $\sigma[w](t)$. Roughly speaking, it is the value of $\sigma[w]$ "just before $t$ ".

The strategy is the following:

$\sigma[w](t)= \begin{cases}\sigma[w]\left(t^{-}\right), & \text {if } q_{\sigma[w]\left(t^{-}\right)}(w(t))<0 \\ \underset{i \in\{1, \ldots, N\}}{\arg \min } q_{i}(w(t)), & \text { if } q_{\sigma[w]\left(t^{-}\right)}(w(t))=0\end{cases}$

This strategy is illustrated by an automaton in Figure 1. The automaton must be understood as follows: while the property $q_{\sigma[w]\left(t^{-}\right)}(w(t))<0$ holds, the mode does not change, whereas if the equality $q_{\sigma[w]\left(t^{-}\right)}(w(t))=0$ holds then there is a transition between the mode where the system was evolving and a new mode. This transition is represented by the arrow on the right of the automaton. To know the new mode where the system has to evolve there is a computation of $\sigma[w](t):=\underset{i \in\{1, \ldots, N\}}{\arg \min } q_{i}(w(t))$.

With Assumption 3.3 at hand the global exponential stability of the argmin switching rule with hysteresis may be stated:

Theorem 3.7: Under Assumption 3.3, system (1) with switching rule (14) is globally exponentially stable. More precisely, letting $V$ and $\alpha$ as in Lemma 3.1, (3) holds along the solutions of (1) with (14).

Remark 3.8: The parameter $\mu$ plays a special feature with this strategy. Indeed, the quadratic function $q_{i}, i=$ $1 \ldots N$, define conic regions

$$
\Omega_{i}:=\left\{x \in \mathbb{R}^{n} \mid q_{i}(x) \leq 0\right\} .
$$

These regions may overlap, and their union is $\mathbb{R}^{n}$ by Assumption 3.3. The switching signal changes of mode when the system trajectory hits the boundary of a region, and the next switching happened only if the trajectory leaves an overlap region. For a geometrical illustration of the hysteresis behavior see Figure 2 in the case of two conic regions $\Omega_{1}$ and $\Omega_{2}$. A question of interest with the hysteresis is the size of the overlap regions. Indeed, larger is the overlap regions larger is the minimum time between two switching times. In (13) the parameter $\mu$ has a direct influence on the size of the overlap region: largest is $\mu$ smaller is the overlap region. Therefore a special care in the value of $\mu$ has to be taken.

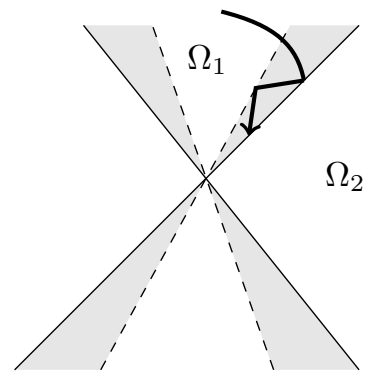

Fig. 2. A possible trajectory for the system. The first conic region $\Omega_{1}$ is in plain line, the second $\Omega_{2}$ is in dashed line. The overlap regions are filled in gray.

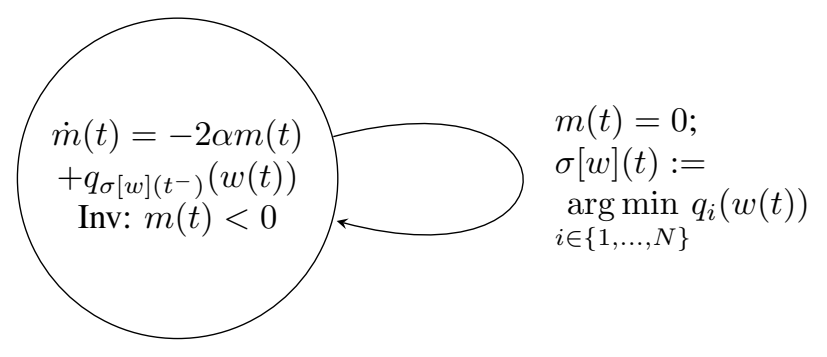

Fig. 3. Argmin, hysteresis and filter automaton.

3) Argmin, hysteresis and filter: Thanks to Lemma 3.1 it holds

$$
\dot{V} \leq-2 \alpha V+q_{\sigma[w](t)}(w(t))
$$

Keeping in mind the objective of decreasing the number of switching times, a low-pass filter is added to the switching rule (14): instead of imposing that $q_{\sigma[w](t)}(w(t)) \leq 0$ at any time $t \geq 0$, we just impose that a weighted averaged value of $q_{\sigma[w](s)}(w(s))$ is negative or zero.

Let us define:

$$
m(t)=e^{-2 \alpha t} \int_{0}^{t} e^{2 \alpha s} q_{\sigma[w](s)}(w(s)) d s, \forall t \leq 0,
$$

where $\alpha$ is the coefficient of decay of the system as in (16). The time derivative of $m$ is given by

$$
\dot{m}(t)=-2 \alpha m(t)+q_{\sigma[w](t)}(w(t)) .
$$

The control consists in keeping $m(t)$ negative or zero at any time (see automaton in Figure 3). The justification for the choice of the function $m$ comes from Gronwall's inequality [see for example [5, pages 708-709]].

Roughly speaking, the rule consists in doing the average of $q_{\sigma[w](s)}(w(s))$ s with different weights given by $e^{\alpha s}$ in the formula. The close past has as much weight in the average than the far past. An explicit description could stated as follow:

$$
\sigma[w](t)= \begin{cases}\sigma[w]\left(t^{-}\right), & \text {if } m(t)<0 \\ \underset{i \in\{1, \ldots, N\}}{\arg \min } q_{i}(w(t)) & \text { if } m(t)=0\end{cases}
$$

So we are able to give our last result of global exponential stability with the above strategy when Assumption 3.3 is satisfied: 
Theorem 3.9: Under Assumption 3.3, system (1) with the switching rule (19) is globally exponentially stable. More precisely, letting $V$ and $\alpha$ as in Lemma 3.1, (3) holds along the solution of (1) with (19).

Remark 3.10: It must be stressed for this last case that the candidate Lyapunov function is not monotonically decreasing contrarily to the two precedent cases. Indeed the strict decrease of $V$ is not obtained. Nonetheless the global exponential stability is proved thanks to the good property of the function $V$.

\section{Simulations}

Let us illustrate our main results by means of numerical simulations. It will illustrate also the interest of the switching strategies when the system of conservation laws (1) is unstable for any constant switching signal $\sigma(t)=\bar{i}$.

Consider the system (1) with two modes $(\mathcal{I}=\{1,2\})$. The initial conditions are selected as the first three elements of an orthonormal basis of $L^{2}([0,1])$. More specifically the following three initial conditions

$$
y_{k}^{0}(x)=\left(\begin{array}{c}
\sqrt{2} \sin ((2 k-1) \pi x) \\
\sqrt{2} \sin (2 k \pi x)
\end{array}\right), k=1,2,3,
$$

are considered. The matrices of the system (1) are $\Lambda_{i}=$ diag $(2,1), i \in\{1,2\}$. Thanks to Remark 2.4 to have boundary matrices $G_{i}$ which destabilize the unswitched system, the matrices must satisfy $\rho\left(\left|G_{i}\right|\right)>1$. It is a necessary condition but not a sufficient one. The instability of the system with the proposed matrices is checked numerically with a Weighted Essentially Non Oscillatory scheme (see [15]). Boundary matrices $G_{1}$ and $G_{2}$ are proposed as

$$
G_{1}=\left(\begin{array}{cc}
1.1 & 0 \\
-0.3 & 0.1
\end{array}\right), \quad G_{2}=\left(\begin{array}{cc}
0 & 0.2 \\
0.1 & -1
\end{array}\right)
$$

The respective spectrum of $\left|G_{1}\right|$ and $\left|G_{2}\right|$ is $\{0.1 ; 1.1\}$ and $\{0.0196 ; 1.0196\}$. See Figure 4 for the constant control input $\bar{i}=1$ and the initial condition $y_{2}^{0}(x)$ where the instability is observed. Firstly the parameter $\mu$ is fixed to the value 0.1 . The Lyapunov matrix $Q$ is set as identity matrix in $\mathbb{R}^{2 \times 2}$. With $\gamma_{1}=\frac{1}{4}$ and $\gamma_{2}=\frac{3}{4}$, it is obtained

$$
\sum_{i=1}^{2} \gamma_{i}\left(G_{i}^{\top} Q \Lambda_{i} G_{i}-e^{-\mu} Q \Lambda_{i}\right)=\left(\begin{array}{ll}
-1.1747 & -0.0825 \\
-0.0825 & -0.0923
\end{array}\right) \text {. }
$$

The eigenvalues of the previous matrix are: -1.1809 and -0.0861 . Therefore it is a symmetric negative definite matrix, and Assumption 3.3 holds.

The three rules proposed in Theorems 3.6, 3.7 and 3.9 stabilize the switched system (1). The time evolution of the two components with the argmin switching rule (11) is shown in Figure 5 with the initial condition $y_{2}^{0}(x)$. The results obtained with the three switching rules are reported in Table I. As it was expected the switching rule (11) is the one which produces larger number of switches by time unit, then it is the switching rule (14) and finally (19).

Up to now the speed of exponential convergence has been fixed, no optimization of $\mu$ has been done. Now, as it was aforementionned in Remark 3.4, the speed can be optimized
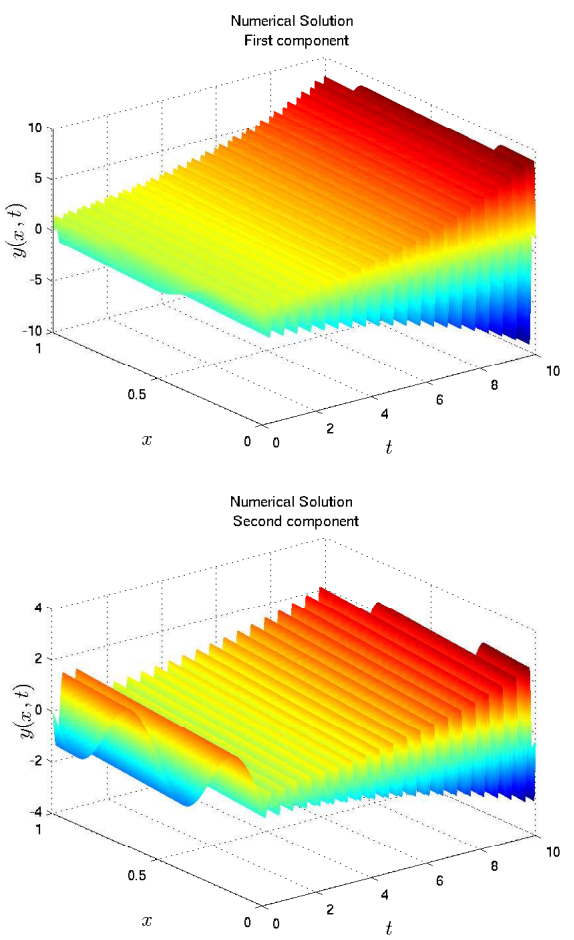

Fig. 4. Time evolution of the first component $y_{1}$ (top) and of the second component $y_{2}$ (bottom) of the solution of the unswitched system (1) with the active mode $\bar{i}=1$ for the example.

by two line searches over $\mu$ and $\gamma_{1}\left(\gamma_{2}\right.$ is related to $\gamma_{1}$ by the relation $\left.\gamma_{2}=1-\gamma_{1}\right)$. Thus it gives successive LMIs solved, e.g. by the Multi-Parametric Toolbox [13]. The algorithm ends at the value $\mu=0.455$. The Lyapunov matrix $Q$ is equal to the identity and $\gamma_{1}$ is found to be equal to 0.45 . One checks that the rule (14) gives more switching times with the optimized $\mu=0.455$ than with $\mu=0.1$, see the third column of Table I. This difference is due to the fact that the intersection of $\Omega_{1}$ and $\Omega_{2}$ (defined by (15)) is tighter with the optimized $\mu=0.455$ than with $\mu=0.1$, in other words the grey domain of Figure 2 is smaller. Furthermore, with argmin switching rule (11) the number of switches per time unit is similar between the two cases, see second column of Table I. Despite the optimization on $\mu$, the speed of convergence computed numerically along the solution to (1) is not better with the two first rules (11) and (14). However the speed of convergence seems to be larger with the switching rule (19) with the optimized $\mu=0.455$ than with $\mu=0.1$.

\section{Conclusion}

In this paper, three switching rules to stabilize hyperbolic systems of conservation laws of the form (1) have been derived. These switching rules correspond to an output feedback law. The analysis of exponential stability is based on Lyapunov techniques. This method has been adapted to the design problem of a stabilizing switching rule. The three switched controllers gave different performances which have been discussed and compared by some numerical simulations. 


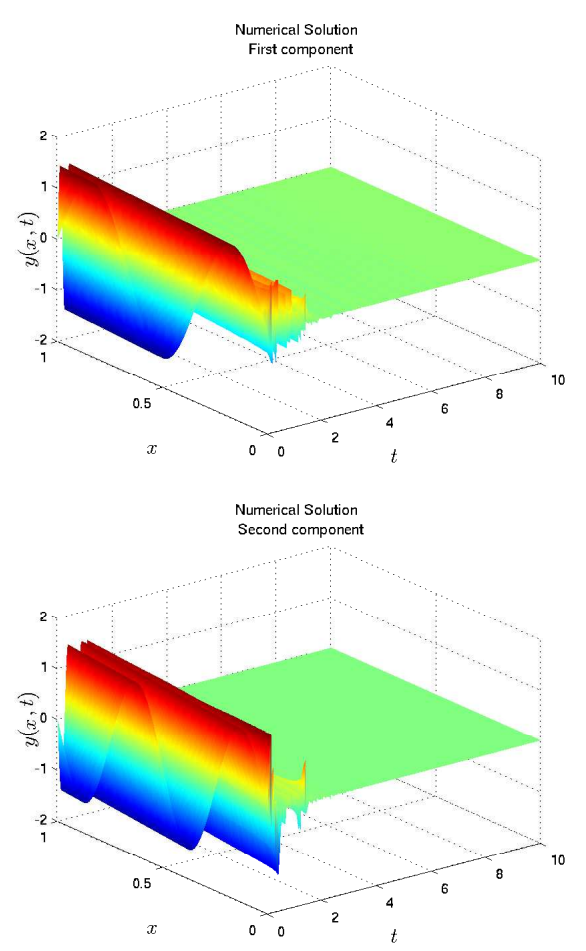

Fig. 5. Time evolution of the first component $y_{1}$ (top) and of the second component $y_{2}$ (bottom) of the solution of (1) with the argmin rule for the example.

\begin{tabular}{|c|c|c|c|}
\hline & Argmin & Hysteresis & Low-pass filter \\
\hline $\begin{array}{l}\text { Initial condition } \\
y_{k}^{0}\end{array}$ & $\begin{array}{l}\text { Exponential } \\
\text { Stability. } \\
\text { Lyapunov } \\
\text { Function. }\end{array}$ & $\begin{array}{l}\text { Exponential } \\
\text { Stability. } \\
\text { Lyapunov } \\
\text { Function. }\end{array}$ & $\begin{array}{l}\text { Exponential } \\
\text { Stability. }\end{array}$ \\
\hline \multicolumn{4}{|c|}{$\begin{array}{l}\text { Theoretical bound on the speed of convergence: } 0.05 \\
\text { (without optimization on } \mu \text { ) }\end{array}$} \\
\hline \multicolumn{4}{|c|}{ Number of switches by time unit. } \\
\hline $\mathrm{k}=1$ & 4.2 & 2.8 & 0.2 \\
\hline $\mathrm{k}=2$ & 21.2 & 13.7 & 0.1 \\
\hline $\mathrm{k}=3$ & 18.5 & 17.1 & 0.1 \\
\hline \multicolumn{4}{|c|}{ Speed of convergence } \\
\hline $\mathrm{k}=1$ & 1.4278 & 1.3393 & 0.0960 \\
\hline $\mathrm{k}=2$ & 1.6332 & 1.5466 & 0.1279 \\
\hline $\mathrm{k}=3$ & 1.4989 & 1.4596 & 0.1209 \\
\hline \multicolumn{4}{|c|}{$\begin{array}{l}\text { Theoretical bound on the speed of convergence: } 0.2275 \\
\text { (with optimization on } \mu \text { ) }\end{array}$} \\
\hline \multicolumn{4}{|c|}{ Number of switches by time unit. } \\
\hline $\mathrm{k}=1$ & 4.2 & 4.2 & 0.4 \\
\hline $\mathrm{k}=2$ & 21.2 & 21.3 & 0.3 \\
\hline $\mathrm{k}=3$ & 18.5 & 18.4 & 0.3 \\
\hline \multicolumn{4}{|c|}{ Speed of convergence } \\
\hline $\mathrm{k}=1$ & 1.4455 & 1.4461 & 0.3851 \\
\hline $\mathrm{k}=2$ & 1.6510 & 1.7648 & 0.3607 \\
\hline $\mathrm{k}=3$ & 1.5166 & 1.5751 & 0.3508 \\
\hline
\end{tabular}

TABLE I

COMPARISON OF THE DIFFERENT SWITCHING STRATEGIES FOR THE EXAMPLE WITH THREE INITIAL CONDITIONS IN $L^{2}([0,1])$ BASIS. PERFORMED DURING 10 UNITS OF TIME.
The first switching rule is based on an argmin computation. It allows to maximize the decay rate of the Lyapunov function at any time. In order to decrease the number of switches in a time interval, a hysteresis strategy to this rule was added. It means that the system stays in the current mode, if it makes the Lyapunov function decrease. In order to reduce again the number of switches a low-pass filter was added to the last rule.

A sufficient condition on the matrices $G_{i}$ and $\Lambda_{i}$ has been derived to get the exponential stability of the system with these three switching rules. Finally, an example was given to illustrate the results.

This work leaves many questions open. In particular, question of existence of solutions has to be investigated when closing the loop with a switching rule. In our analysis we have supposed that $y$ lies in $C_{p w}([0,1])$ but this assumption was not checked with the three rules (but observed only numerically). Furthermore the overshoot reduction, or time performance may be tackled in a future work.

\section{REFERENCES}

[1] S. Amin, F. M. Hante, and A. M. Bayen. Exponential stability of switched linear hyperbolic initial-boundary value problems. IEEE Trans. Automat. Contr., 57(2):291-301, 2012.

[2] J.-M. Coron, G. Bastin, and B. d'Andréa Novel. Dissipative boundary conditions for one-dimensional nonlinear hyperbolic systems. SIAM J. Control Optim., 47(3):1460-1498, may 2008.

[3] A. Diagne, G. Bastin, and J.-M. Coron. Lyapunov exponential stability of 1-d linear hyperbolic systems of balance laws. Automatica, 48:109114, jan 2012.

[4] V. Dos Santos and C. Prieur. Boundary control of open channels with numerical and experimental validations. IEEE Trans. on Control Systems Technology, 16(6):1252 -1264, nov. 2008.

[5] L.C. Evans. Partial Differential Equations. Graduate Studies in Mathematics. American Mathematical Society, 2010.

[6] J. C. Geromel and P. Colaneri. Stability and stabilization of discrete time switched systems. International Journal of Control, 79(7):719728, 2006.

[7] M. Gugat, M. Dick, and G. Leugering. Gas flow in fan-shaped networks: Classical solutions and feedback stabilization. SIAM Journal on Control and Optimization, 49(5):2101-2117, 2011.

[8] Martin Gugat and Mario Sigalotti. Stars of vibrating strings: switching boundary feedback stabilization. Networks and Heterogeneous Media, 5(2):299-314, 2010.

[9] F. M. Hante, G. Leugering, and T. I. Seidman. Modeling and analysis of modal switching in networked transport systems. Appl. Math. Optim., 59(2):275-292, 2009.

[10] F. M. Hante, G. Leugering, and T. I. Seidman. An augmented BV setting for feedback switching control. Journal of Systems Science and Complexity, 23(3):456-466, 2010.

[11] F. M. Hante and M. Sigalotti. Converse Lyapunov theorems for switched systems in Banach and Hilbert spaces. SIAM J. Control Optim., 49(2):752-770, 2011.

[12] B. Haut and G. Bastin. A second order model of road junctions in fluid models of traffic networks. Networks and Heterogeneous Media, 2(2):227-257, june 2007

[13] M. Kvasnica, P. Grieder, and M. Baotić. Multi-Parametric Toolbox (MPT), 2004

[14] D. Liberzon. Switching in systems and control. Springer, 2003.

[15] X.-D. Liu, S. Osher, and T. Chan. Weighted essentially non-oscillatory schemes. J. Comput. Phys., 115(1):200-212, nov. 1994.

[16] C. Prieur, A. Girard, and E. Witrant. Lyapunov functions for switched linear hyperbolic systems. 4th IFAC Conference on Analysis and Design of Hybrid Systems (ADHS'12), 2012. Eindhoven, The Netherlands. 